\title{
Rescheduling Problems with Agreeable Job Parameters to Minimize the Tardiness Costs under Deterioration and Disruption
}

\author{
Zhang Xingong ${ }^{1,2}$ and Wang Yong ${ }^{1}$ \\ ${ }^{1}$ College of Economics and Business Administration, Chongqing University, Chongqing 400030, China \\ ${ }^{2}$ College of Mathematical Sciences, Chongqing Normal University, Chongqing 400047, China \\ Correspondence should be addressed to Wang Yong; wangyongcq@126.com
}

Received 21 May 2013; Accepted 18 July 2013

Academic Editor: Yunqiang Yin

Copyright ( $) 2013$ Z. Xingong and W. Yong. This is an open access article distributed under the Creative Commons Attribution License, which permits unrestricted use, distribution, and reproduction in any medium, provided the original work is properly cited.

\begin{abstract}
This paper considers single-machine rescheduling problems with agreeable job parameters under deterioration and disruption. Deteriorating jobs mean that the processing time of a job is defined by an increasing function of its starting time. Rescheduling means that, after a set of original jobs has already been scheduled, a new set of jobs arrives and creates a disruption. We consider four cases of minimization of the total tardiness costs with agreeable job parameters under a limit of the disruptions from the original job sequence. We propose polynomial-time algorithms or some dynamic programming algorithms under sequence disruption and time disruption.
\end{abstract}

\section{Introduction}

Scheduling problems are very important in manufacturing systems. Hence, numerous scheduling problems have been studied for many years. In the classical scheduling theory, job processing times are assumed to be known and fixed from the first job to be processed until the last job to be completed. However, there are many situations in which a job that is processed later consumes more time than the same job when processed earlier. Scheduling in this setting is known as scheduling deteriorating jobs.

Significant contributions towards addressing or solving deteriorating job scheduling problems on a single machine include, among others, the following: Browne and Yechiali [1] cited applications concerning the control of queues and communication systems where jobs deteriorate as they await processing. Kunnathur and Gupta [2] and Mosheiov [3] gave several other real-life situations where deteriorating jobs occur. These include the search for an object under worsening weather or performance of medical treatments under deteriorating health conditions. Comprehensive discussion of scheduling problems with time-dependent processing times of jobs can be found in Cheng et al. [4] and Gawiejnowicz [5]. Recently, Biskup and Herrmann [6] observed that the sum of the processing times of the jobs processed before a job contributes to the actual processing time of the job, and they cite equipment wearout (e.g., a drill) as a real-life example of their observation. Wang and Guo [7] considered a single-machine scheduling problem with the effects of learning and deterioration. The goal is to determine an optimal combination of the due date and schedule so as to minimize the sum of earliness, tardiness, and due-date costs. $\mathrm{Ng}$ et al. [8] considered a two-machine flow shop scheduling problem with linearly deteriorating jobs to minimize the total completion time. Cheng et al. [9] considered scheduling with deteriorating jobs in which the actual processing time of a job is a function of the logarithm of the total processing time of the jobs processed before it (to avoid the unrealistic situation where the jobs scheduled lately will incur excessively long processing times), and the setup times are proportional to the actual processing times of the already scheduled jobs. 
Rescheduling involves adjusting a previously planned, possibly optimal, scheduling to account for a disruption. Examples of common disruptions include: the arrival of new orders, order cancelations, changes in order priority, processing delays, changes in release dates, machine breakdowns, and the unavailability of raw materials, personnel, or tools. There are several papers on rescheduling approaches in manufacturing systems. Raman et al. [10] developed a branch-and-bound procedure to reschedule a flexible manufacturing system in the presence of dynamic job arrivals. Church and Uzsoy [11] addressed a similar problem for which they describe periodic rescheduling policies and analyze their error bounds. Jain and Elmaraghy [12] used genetic algorithms to develop heuristic approaches for rescheduling a flexible manufacturing system. Vieira et al. [13] provided an extensive review of rescheduling problems. Yang [14] studied the single-machine rescheduling with new jobs arrivals and processing time compression. Hall and Potts [15] considered the problem of rescheduling of a single machine with newly arrived jobs to minimize the maximum lateness and the total completion time under a limit of the disruption from the original scheduling. Yuan and $\mathrm{Mu}$ [16] considered the rescheduling problem for jobs on a single machine with release dates to minimize makespan under a limit on the maximum sequence disruption. Zhao and Tang [17] presented two single-machine rescheduling problems with linear deteriorating jobs under disruption. deteriorating jobs mean that the actual processing time of the job is an increasing function of its starting time. They considered the rescheduling problem to minimize the total completion time under a limit of the disruption from the original scheduling. Hoogeveen et al. [18] tackled several simple setup time configurations yielding different scheduling problems for which they propose optimal polynomial time algorithms or provide NP-hardness proofs. They also present the problem of enumerating the set of strict Pareto optima for the sum of setup times and disruption cost criteria.

Based on the motivation of Hall and Potts [15] and Zhao and Tang [17], we consider some rescheduling problems with the criterion minimizing the total tardiness costs under a limit of the disruption from the original schedule in this paper. The rest of the paper is organized as follows. In the next section, we give the problem description. In Section 3, we consider single-machine scheduling problems. The last section is the conclusion.

\section{Problem Definition and Notation}

By the terminology of Hall and Potts [15], our researchful problem can be stated as follows. Let $J_{0}=\left\{J_{1}, \ldots, J_{n_{0}}\right\}$ denote a set of original jobs to be processed non preemptively on a single machine. In the presented model, we assume that these jobs have been scheduled optimally to minimize some classical objective and that $\pi^{*}$ is an optimal job sequence with no idle time between the jobs. Let $J_{N}=\left\{J_{n_{0}+1}, \ldots, J_{n_{0}+n_{N}}\right\}$ denote a set of new jobs that arrive together. We assume that these jobs arrive at time zero after a schedule for the jobs of $J_{0}$ has been determined, but before processing begins. There is no loss of generality in this assumption: if the jobs arrive after time zero, then the fully processed jobs of $J_{0}$ are removed from the problem; any partly processed jobs are processed to completion, and $J_{0}$ and $n_{0}$ are updated accordingly. Let $J=J_{0} \bigcup J_{N}$ and $n=n_{0}+n_{N}$. Each job $J_{j} \in J$ has an integral normal processing time $p_{j}$ and a deteriorating rate $b>0$; the actual processing time of job $J_{j}$ is $p_{j}\left(a+b s_{j}\right)$, where $s_{j}(\geq 0)$ is the starting time of job $J_{j}$ and $a(>0)$ is constant. For any schedule $\sigma$ of the jobs in $J$, we define the following variables:

$s_{j}(\sigma)$ is the time at which job $J_{j} \in J$ starts its processing in schedule $\sigma$.

$d_{j}(\sigma)$ is the due date of job $J_{j} \in J$ in schedule $\sigma$.

$C_{j}(\sigma)$ is the time at which job $J_{j} \in J$ is completed in schedule $\sigma$.

$T_{j}(\sigma)=\max \left\{C_{j}(\sigma)-d_{j}, 0\right\}$ is the tardiness value of job $J_{j} \in J$.

$D_{j}\left(\pi^{*} ; \sigma\right)$ is the sequence disruptions of job $J_{j} \in J_{0}$; that is, if $J_{j}$ is the $x$ th job in $\pi^{*}$ and the $y$ th job in $\sigma$, respectively, then $D_{j}\left(\pi^{*} ; \sigma\right)=|y-x|$.

$\Delta_{j}\left(\pi^{*} ; \sigma\right)=\left|C_{j}(\sigma)-C_{j}\left(\pi^{*}\right)\right|$ is the time disruption of job $J_{j} \in J_{0}$.

When there is no ambiguity, we simplify the above symbols and write $s_{j}, d_{j} C_{j}, T_{j}, D_{j}\left(\pi^{*}\right)$, and $\Delta_{j}\left(\pi^{*}\right)$, respectively.

We consider only the single-scheduling problems with the following constraints on the amount of disruption, where $k \geq 0$ is a known integer:

$D_{\max }\left(\pi^{*}\right) \leq k: \max \left\{D_{j}\left(\pi^{*}\right) J_{j} \in J_{0}\right\} \leq k$; the maximum sequence disruption of the jobs cannot exceed $k$.

$\sum D_{j}\left(\pi^{*}\right) \leq k: \sum_{J_{j} \in J_{0}} D_{j}\left(\pi^{*}\right) \leq k$; the total sequence disruption of the jobs cannot exceed $k$.

$\Delta_{\max }\left(\pi^{*}\right) \leq k: \max \left\{\Delta_{j}\left(\pi^{*}\right) J_{j} \in J_{0}\right\} \leq k$; the maximum time disruption of the jobs cannot exceed $k$.

$\sum \Delta_{j}\left(\pi^{*}\right) \leq k: \sum_{J_{j} \in J_{0}} \Delta_{j}\left(\pi^{*}\right) \leq k$; the total time disruption of the jobs cannot exceed $k$.

Since the $1 \mid \sum T_{j}$ problem is NP-hard in Du and Leung [19], the $1|\Gamma \leq k| \sum T_{j}$ problem is also NP-hard, where $\Gamma \epsilon$ $\left\{D_{\max }\left(\pi^{*}\right), \sum D_{j}\left(\pi^{*}\right), \Delta_{\max }\left(\pi^{*}\right), \sum \Delta_{j}\left(\pi^{*}\right)\right\}$. In this paper, we consider a special case: the processing time and due date of jobs are agreeable; that is, $p_{i} \leq p_{j} \Rightarrow d_{i} \leq d_{j}$ for all jobs $J_{i}$ and $J_{j}$.

Using the three-field notation [20], the considered problems can be denoted as

$1\left|p_{i} \leq p_{j} \Rightarrow d_{i} \leq d_{j}, D_{\max }\left(\pi^{*}\right) \leq k, p_{j}\left(a+b s_{j}\right)\right|$

$\sum T_{j}$

$1\left|p_{i} \leq p_{j} \Rightarrow d_{i} \leq d_{j}, \sum D_{j}\left(\pi^{*}\right) \leq k, p_{j}\left(a+b s_{j}\right)\right|$

$\sum T_{j}$;

$1\left|p_{i} \leq p_{j} \Rightarrow d_{i} \leq d_{j}, \Delta_{\max }\left(\pi^{*}\right) \leq k, p_{j}\left(a+b s_{j}\right)\right|$

$\sum T_{j}$;

$1\left|p_{i} \leq p_{j} \Rightarrow d_{i} \leq d_{j}, \sum \Delta_{j}\left(\pi^{*}\right) \leq k, p_{j}\left(a+b s_{j}\right)\right|$ $\sum T_{j}$. 


\section{Minimum Tardiness Problem with Agreeable Job Parameters}

We start with the following result by Kononov and Gawiejnowicz [21].

Lemma 1. For the $1\left|p_{j}\left(a+b s_{j}\right)\right| C_{\max }$ problem, if $\pi=\left\{J_{1}, \ldots, J_{n}\right\}$ and the starting time of the job $J_{1}$ is $t$, then makespan is sequence independent, and

$$
C_{\max }(\pi)=t \prod_{i=1}^{n}\left(1+b p_{i}\right)+\frac{a}{b}\left(\prod_{i=1}^{n}\left(1+b p_{i}\right)-1\right) .
$$

For notational convenience, we assume that the jobs are indexed by agreeable order; that is, $p_{1} \leq \cdots \leq p_{n_{0}}$ and $d_{1} \leq$ $\cdots \leq d_{n_{0}}$. Thus, $\pi^{*}=\left\{J_{1}, \ldots, J_{n_{0}}\right\}$ with no idle time between jobs. We now show that the EDD or SPT rule applies to serval of the rescheduling problems we consider.

Lemma 2. Problems $1 \mid p_{i} \leq p_{j} \Rightarrow d_{i} \leq d_{j}, D_{\max }\left(\pi^{*}\right) \leq$ $k, p_{j}\left(a+b s_{j}\right) \mid \sum T_{j}$, and $1 \mid p_{i} \leq p_{j} \Rightarrow d_{i} \leq d_{j}, \Delta_{\max }\left(\pi^{*}\right) \leq$ $k, p_{j}\left(a+b s_{j}\right) \mid \sum T_{j}$ have an optimal schedule with no idle time between jobs, and

(a) a schedule for problem $1 \mid p_{i} \leq p_{j} \Rightarrow d_{i} \leq d_{j}$, $D_{\max }\left(\pi^{*}\right) \leq k, p_{j}\left(a+b s_{j}\right) \mid \sum T_{j}$ is feasible if the number of jobs of $J_{N}$ scheduled before the last job of $J_{0}$ is less than or equal to $k$;

(b) a schedule for problem $1 \mid p_{i} \leq p_{j} \Rightarrow d_{i} \leq d_{j}$, $\Delta_{\max }\left(\pi^{*}\right) \leq k, p_{j}\left(a+b s_{j}\right) \mid \sum T_{j}$ is feasible if the total actual processing time of jobs of $J_{N}$ scheduled before the last job of $J_{0}$ is less than or equal to $k$.

Proof. The proof is similar to that of Lemma 1 in Hall and Potts [15].

Lemma 3. For problems $1 \mid p_{i} \leq p_{j} \Rightarrow d_{i} \leq d_{j}, \Gamma \leq k$ and $p_{j}\left(a+b s_{j}\right) \mid \sum T_{j}$, where $\Gamma \in\left\{D_{\max }\left(\pi^{*}\right), \sum D_{j}\left(\pi^{*}\right)\right.$, $\left.\Delta_{\max }\left(\pi^{*}\right), \sum \Delta_{j}\left(\pi^{*}\right)\right\}$, there exists an optimal schedule in which the jobs of $J_{0}$ are sequenced in the EDD or SPT rule as in $\pi^{*}$, the jobs of $J_{N}$ are sequenced in the EDD or SPT rule, and there is no idle time between jobs.

Proof. We first analyze the jobs of $J_{0}$. Consider an optimal schedule $\sigma^{*}$ in which the jobs of $J_{0}$ are not sequenced in the EDD or SPT rule as in $\pi^{*}$. Let $J_{i}$ be the job with the smallest index that appears later relative to the other jobs of $J_{0}$ in $\sigma^{*}$ than in $\pi^{*}$, and let $J_{j}(j>i)$ be the last job of $J_{0}$ that precedes job $J_{i}$ in $\sigma^{*}$. Because $\pi^{*}$ is an optimal sequence, $p_{j}$ and $d_{j}$ are agreeable. Assume that the starting time of job $J_{j}$ in $\sigma^{*}$ is $t_{0}$, then $C_{j}\left(\sigma^{*}\right)=t_{0}+p_{j}\left(a+b t_{0}\right)$. Perform an interchange on jobs $J_{j}$ and $J_{i}$, and get a new schedule $\sigma^{\prime}$. In $\sigma^{\prime}$, the starting time of job $J_{i}$ is $t_{0}$, then $C_{i}\left(\sigma^{\prime}\right)=t_{0}+p_{i}\left(a+b t_{0}\right)<$ $t_{0}+p_{j}\left(a+b t_{0}\right)=C_{j}\left(\sigma^{*}\right)$. From Lemma $1, C_{j}\left(\sigma^{\prime}\right)=C_{i}\left(\sigma^{*}\right)$. Thus, the jobs between job $J_{i}$ and $J_{j}$ are completed earlier in $\sigma^{\prime}$ than in $\sigma^{*}$. Next, we consider the total tardiness of jobs $J_{i}$ and $J_{j}$ in $\sigma^{\prime}$ and in $\sigma^{*}$.
The total tardiness of jobs $J_{i}$ and $J_{j}$ in $\sigma^{*}$ is as follows:

$$
\begin{aligned}
T_{i}\left(\sigma^{*}\right)+T_{j}\left(\sigma^{*}\right)= & \max \left\{C_{i}\left(\sigma^{*}\right)-d_{i}, 0\right\} \\
& +\max \left\{C_{j}\left(\sigma^{*}\right)-d_{j}, 0\right\} .
\end{aligned}
$$

The total tardiness of jobs $J_{i}$ and $J_{j}$ in $\sigma^{\prime}$ is as follows:

$$
\begin{aligned}
T_{i}\left(\sigma^{\prime}\right)+T_{j}\left(\sigma^{\prime}\right)= & \max \left\{C_{i}\left(\sigma^{\prime}\right)-d_{i}, 0\right\} \\
& +\max \left\{C_{j}\left(\sigma^{\prime}\right)-d_{j}, 0\right\} .
\end{aligned}
$$

To compare the total tardiness of jobs $J_{i}$ and $J_{j}$ in $\sigma^{*}$ and in $\sigma^{\prime}$, we divide it into two cases. In the first case, when $C_{j}\left(\sigma^{*}\right) \leq d_{j}$, we have $T_{i}\left(\sigma^{*}\right)+T_{j}\left(\sigma^{*}\right)=\max \left\{C_{i}\left(\sigma^{*}\right)-d_{i}, 0\right\}$. Suppose that neither $T_{i}\left(\sigma^{\prime}\right)$ nor $T_{j}\left(\sigma^{\prime}\right)$ is zero. Note that this is the most restrictive case since it comprises the case that either one or both $T_{i}\left(\sigma^{\prime}\right)$ and $T_{j}\left(\sigma^{\prime}\right)$ are zero. From Lemma 1 and $p_{i} \leq p_{j}, d_{i} \leq d_{j}$, we have $\left\{T_{i}\left(\sigma^{*}\right)+T_{j}\left(\sigma^{*}\right)\right\}-\left\{T_{i}\left(\sigma^{\prime}\right)+T_{j}\left(\sigma^{\prime}\right)\right\}=$ $C_{i}\left(\sigma^{*}\right)-C_{i}\left(\sigma^{\prime}\right)-C_{j}\left(\sigma^{\prime}\right)+d_{j}=d_{j}-C_{i}\left(\sigma^{\prime}\right) \geq d_{j}-C_{j}\left(\sigma^{*}\right) \geq 0$.

In the second case, when $C_{j}\left(\sigma^{*}\right)>d_{j}$, we have $T_{i}\left(\sigma^{*}\right)+$ $T_{j}\left(\sigma^{*}\right)=C_{i}\left(\sigma^{*}\right)+C_{j}\left(\sigma^{*}\right)-d_{i}-d_{i}$. Suppose that neither $T_{i}\left(\sigma^{\prime}\right)$ nor $T_{j}\left(\sigma^{\prime}\right)$ is zero. From Lemma 1 and $p_{i} \leq p_{j}, d_{i} \leq d_{j}$, we have

$$
\begin{aligned}
& \left\{T_{i}\left(\sigma^{*}\right)+T_{j}\left(\sigma^{*}\right)\right\}-\left\{T_{i}\left(\sigma^{\prime}\right)+T_{j}\left(\sigma^{\prime}\right)\right\} \\
& \quad=C_{i}\left(\sigma^{*}\right)+C_{j}\left(\sigma^{*}\right)-C_{j}\left(\sigma^{\prime}\right)-C_{i}\left(\sigma^{\prime}\right) \geq 0 .
\end{aligned}
$$

Now, we have proved that the total tardiness of $\sigma^{\prime}$ is less than or equal to that of $\sigma^{*}$.

Let the position of job $J_{i}$ in $\pi^{*}$ be $k_{1}$ and let the position of job $J_{j}$ in $\pi^{*}$ be $k_{2}$ and in $\sigma^{\prime}$ be $k_{3}$. If $k_{3} \geq k_{2}$, then $D_{j}\left(\pi^{*}, \sigma^{\prime}\right)=k_{3}-k_{2}, D_{i}\left(\pi^{*}, \sigma^{*}\right)=k_{3}-k_{1}$. Since $i<j$ implies $k_{1}<k_{2}$, we have $D_{j}\left(\pi^{*}, \sigma^{\prime}\right)<D_{i}\left(\pi^{*}, \sigma^{*}\right)$. If $k_{3}<$ $k_{2}$, then $D_{j}\left(\pi^{*}, \sigma^{\prime}\right)=k_{2}-k_{3}$ and $D_{j}\left(\pi^{*}, \sigma^{*}\right)=k_{2}-$ $\left(k_{3}-h\right)$, where $h$ is the difference between the position of job $J_{i}$ and $J_{j}$ in $\sigma^{*}$. So, $D_{j}\left(\pi^{*}, \sigma^{\prime}\right)<D_{j}\left(\pi^{*}, \sigma^{*}\right)$. Hence, we have $D_{\max }\left(\pi^{*}, \sigma^{\prime}\right)<D_{\max }\left(\pi^{*}, \sigma^{*}\right)$. In either case, because $D_{i}\left(\pi^{*}, \sigma^{\prime}\right)=D_{i}\left(\pi^{*}, \sigma^{*}\right)-h$ and $D_{j}\left(\pi^{*}, \sigma^{\prime}\right) \leq D_{j}\left(\pi^{*}, \sigma^{*}\right)+h$. Hence, $\sum D_{j}\left(\pi^{*}, \sigma^{\prime}\right) \leq \sum D_{j}\left(\pi^{*}, \sigma^{*}\right)$.

Moreover, if $C_{j}\left(\sigma^{\prime}\right) \geq C_{j}\left(\pi^{*}\right)$, then $\Delta_{j}\left(\pi^{*}, \sigma^{\prime}\right)=C_{j}\left(\sigma^{\prime}\right)-$ $C_{j}\left(\pi^{*}\right)$. Since $\Delta_{i}\left(\pi^{*}, \sigma^{*}\right)=C_{i}\left(\sigma^{*}\right)-C_{i}\left(\pi^{*}\right)=C_{j}\left(\sigma^{\prime}\right)-C_{i}\left(\pi^{*}\right)$ and $C_{i}\left(\pi^{*}\right)<C_{j}\left(\pi^{*}\right)$; therefore, $\Delta_{j}\left(\pi^{*}, \sigma^{\prime}\right)<\Delta_{i}\left(\pi^{*}, \sigma^{*}\right)$. If $C_{j}\left(\sigma^{\prime}\right)<C_{j}\left(\pi^{*}\right)$, then $\Delta_{j}\left(\pi^{*}, \sigma^{\prime}\right)=C_{j}\left(\pi^{*}\right)-C_{j}\left(\sigma^{\prime}\right)$ because $\Delta_{j}\left(\pi^{*}, \sigma^{*}\right)=C_{j}\left(\pi^{*}\right)-C_{j}\left(\sigma^{*}\right), \Delta_{j}\left(\pi^{*}, \sigma^{\prime}\right)<\Delta_{j}\left(\pi^{*}, \sigma^{*}\right)$. Thus, we have $\Delta_{\max }\left(\pi^{*}, \sigma^{\prime}\right)<\Delta_{\max }\left(\pi^{*}, \sigma^{*}\right)$. In either case, because $\Delta_{i}\left(\pi^{*}, \sigma^{\prime}\right)=\Delta_{i}\left(\pi^{*}, \sigma^{*}\right)-h^{\prime}$ and $\Delta_{j}\left(\pi^{*}, \sigma^{\prime}\right) \leq$ $\Delta_{j}\left(\pi^{*}, \sigma^{*}\right)+h^{\prime}$, where $h^{\prime}=C_{i}\left(\sigma^{*}\right)-C_{i}\left(\sigma^{\prime}\right)$. Then we deduce that $\sum \Delta_{j}\left(\pi^{*}, \sigma^{\prime}\right) \leq \sum \Delta_{j}\left(\pi^{*}, \sigma^{*}\right)$. Thus, for either problem, $\sigma^{\prime}$ is feasible and optimal. We can show that there exists an optimal schedule in which the jobs of $J_{0}$ are sequenced in the EDD or SPT order as in $\pi^{*}$ by finite numbers of repetitions of the argument. A similar interchange argument establishes 
that the jobs of $J_{N}$ can also be obtained by sequencing in the EDD or SPT order. The same EDD or SPT ordering of the jobs of $J_{0}$ in $\pi^{*}$ and an optimal schedule show that there is no idle time in this optimal schedule. Otherwise, removing this idle time maintains feasibility and decreases the total tardiness.

We refer to the (EDD, EDD) property when a schedule is constructed using Lemmas 2 and 3 . We first consider problem $1\left|p_{i} \leq p_{j} \Rightarrow d_{i} \leq d_{j}, D_{\max }\left(\pi^{*}\right) \leq k, p_{j}\left(a+b s_{j}\right)\right| \sum T_{j}$. From Lemmas 2 and 3 , there are at most $k$ jobs of $J_{N}$ that can be sequenced before the last job of $J_{0}$, and these jobs have the smallest due dates.Thus, we propose the following algorithm under the maximum sequence disruption constraint. (see Box 1).

Algorithm 4. Consider the following steps.

Step 1. Index the job of $J_{N}$ in the EDD order.

Step 2. Schedule jobs $1, \ldots, n_{0}+k$ in the EDD rule in the first $n_{0}+k$ position and schedule jobs $n_{0}+k+1, \ldots, n_{0}+n_{N}$ in the EDD order in the final $n_{N}-k$ positions.

Theorem 5. For the $1 \mid p_{i} \leq p_{j} \Rightarrow d_{i} \leq d_{j}, D_{\max }\left(\pi^{*}\right) \leq$ $k, p_{j}\left(a+b s_{j}\right) \mid \sum T_{j}$ problem, Algorithm 4 finds an optimal schedule in $O\left(n+n_{N} \log n_{N}\right)$ time.

Proof. From Lemmas 2 and 3, the constraint $D_{\max }\left(\pi^{*}\right) \leq k$ allows at most $k$ jobs of $J_{N}$ to be sequenced before the final of $J_{0}$, and these are the jobs of $J_{N}$ with the smallest due dates. Classical schedule theory shows that the jobs of this first group are sequenced in the EDD order, while Lemma 3 establishes that the remaining $n_{N}-k$ jobs of $J_{N}$ are also sequenced in the EDD order.

Next, we note that the Step 1 for the jobs of $J_{N}$ requires $O\left(n_{N} \log n_{N}\right)$ time. Step 2 is executed in $O(n)$ time by merging the first $k$ jobs of the EDD ordered jobs of $J_{N}$ with the jobs of $J_{0}$ as sequenced in $\pi^{*}$ and then placing the last $n_{N}-k$ jobs of the EDD order ordered jobs of $J_{N}$ at the end of the schedule.

Next, we consider problem $1 \mid p_{i} \leq p_{j} \Rightarrow d_{i} \leq$ $d_{j}, \sum D_{j}\left(\pi^{*}\right) \leq k, p_{j}\left(a+b s_{j}\right) \mid \sum T_{j}$. From Lemmas 1, 2, and 3 , there is the total sequence disruption of the jobs of $J_{N}$ which is less than or equal to $k$ and can be sequenced before the last job of $J_{0}$, and these jobs have the smallest due dates. Thus, we propose the following algorithm under the total sequence disruption constraint. (see Box 2).

Let $f(i, j, \delta)$ be minimum total tardiness value of a partial schedule for jobs $J_{1}, \ldots, J_{i}$ and $J_{n_{0}+1}, \ldots, J_{n_{0}+j}$, where the total sequence disruption is equal to $\delta$. The dynamic programming procedure can now be stated as follows.

Algorithm 6. Consider the following steps.

Step 1 (Initialization). Set $f(i, j, \delta)=0$ for $(i, j, \delta)=(0,0,0)$ and $f(i, j, \delta)=\infty$ for $(i, j, \delta) \neq(0,0,0), i=1, \ldots, n_{0}, j=$ $1, \ldots, n_{N}, i=0, \ldots, k$.
Step 2 (Recurrence Relation).

$$
f(i, j, \delta)=\min \left\{\begin{array}{c}
f(i-1, j, \delta-j)+\max \left\{C_{i}-d_{i}, 0\right\} ; \\
f(i, j-1, \delta)+\max \left\{C_{n_{0}+j}-d_{n_{0}+j}, 0\right\},
\end{array}\right.
$$

where $C_{j}$ denotes the completion time of job $J_{j}$.

Step 3 (Optimal Solution). Calculate the optimal solution value $\min _{0 \leq \delta \leq k}\left\{f\left(n_{0}, n_{N}, \delta\right)\right\}$.

In the recurrence relation, the first term in the minimization corresponds to the case where the partial schedule ends with job $J_{i} \in J_{0}$. Because $J_{j}$ jobs of $J_{N}$ appear before job $J_{i}$ in such a partial schedule, the increase in total sequence disruption is equal to $j$. The second term corresponds to the case where the partial schedule ends with job $J_{n_{0}+j} \in J_{N}$.

In addition, we demonstrate the result of Algorithm 6 in the following example.

Example 7. $n_{0}=3, n_{N}=3, t_{0}=0, J_{0}=\left\{J_{1}, J_{2}, J_{3}\right\}, J_{N}=$ $\left\{J_{4}, J_{5}, J_{6}\right\}, p_{1}=3.2, d_{1}=4, p_{2}=1, d_{2}=2.4, p_{3}=2, d_{3}=$ $3, p_{4}=1.5, d_{4}=3, p_{5}=4, d_{5}=5, p_{6}=3, d_{6}=4.7, a=0.2$, $b=0.4$, and $k=5$.

Solution: According to Algorithm 4 and Lemmas 2 and 3. Because the total sequence disruption of the jobs $J_{1}, J_{2}, J_{3}$ can not exceed $k=5$. By dynamic programming algorithm, we obtain job sequence and the total tardiness cost as follows:

if $k=0$, the optimal sequence is $\left[J_{2} \rightarrow J_{3} \rightarrow\right.$ $J_{1} \rightarrow J_{4} \rightarrow J_{6} \rightarrow J_{5}$ ], and the total tardiness cost is 26.8007;

if $k=0$, the job sequence is $\left[J_{2} \rightarrow J_{3} \rightarrow J_{1} \rightarrow\right.$ $J_{4} \rightarrow J_{6} \rightarrow J_{5}$, and the total tardiness cost is 26.8007;

if $k=1$, the job sequence is $\left[J_{2} \rightarrow J_{3} \rightarrow J_{4} \rightarrow\right.$ $J_{1} \rightarrow J_{6} \rightarrow J_{5}$, and the total tardiness cost is 25.8007;

if $k=2$, the job sequence is $\left[J_{2} \rightarrow J_{4} \rightarrow J_{3} \rightarrow\right.$ $J_{1} \rightarrow J_{6} \rightarrow J_{5}$, and the total tardiness cost is 25.8007;

if $k=3$, the job sequence is $\left[J_{4} \rightarrow J_{2} \rightarrow J_{3} \rightarrow\right.$ $J_{1} \rightarrow J_{6} \rightarrow J_{5}$, and the total tardiness cost is 25.8007;

if $k=4$, the job sequence is $\left[J_{4} \rightarrow J_{2} \rightarrow J_{3} \rightarrow\right.$ $J_{6} \rightarrow J_{1} \rightarrow J_{5}$, and the total tardiness cost is 26.8223;

if $k=5$, the job sequence is $\left[J_{4} \rightarrow J_{2} \rightarrow J_{6} \rightarrow\right.$ $J_{3} \rightarrow J_{1} \rightarrow J_{5}$, and the total tardiness cost is 28.5223

Furthermore, if total sequence disruption $k$ is equal to $1,2,3$, then minimizing total tardiness cost is 25.8007 , and optimal job sequence is $\left[J_{2} \rightarrow J_{3} \rightarrow J_{4} \rightarrow J_{1} \rightarrow J_{6} \rightarrow J_{5}\right.$, $\left[J_{2} \rightarrow J_{4} \rightarrow J_{3} \rightarrow J_{1} \rightarrow J_{6} \rightarrow J_{5}\right]$, and $\left[J_{4} \rightarrow J_{2} \rightarrow\right.$ $\left.J_{3} \rightarrow J_{1} \rightarrow J_{6} \rightarrow J_{5}\right]$. 
Input: Given $p_{j}, d_{j}$ for $j=1, \ldots, n ; k$ and $\pi^{*}$, where $k \leq n_{N}$.

Indexing: Index the jobs of $J_{N}$ in the EDD order.

Schedule Construction: Schedule jobs $1, \ldots, n_{0}+k$ in the EDD order in the first $n_{0}+k$ positions.

Schedule jobs $n_{0}+k+1, \ldots, n_{0}+n_{N}$ in EDD order in the final $n_{N}-k$ positions.

Box 1

Input: Given $p_{j}, d_{j}$ for $j=1, \ldots, n ; k$ and $\pi^{*}$, where $k \leq n_{0} n_{N}$.

Indexing: Index the jobs of $J_{N}$ in the EDD order.

Box 2

Theorem 8. For the $1 \mid p_{i} \leq p_{j} \Rightarrow d_{i} \leq d_{j}, \sum D_{j}\left(\pi^{*}\right) \leq$ $k, p_{j}\left(a+b s_{j}\right) \mid \sum T_{j}$ problem, Algorithm 6 finds an optimal schedule in $\mathrm{O}\left(n_{0}^{2} n_{N}^{2}\right)$ time.

Proof. From Lemmas 1, 2, and 3, it only remains to enumerate all possible ways of merging the EDD ordered lists of jobs of $J_{0}$ and $J_{N}$. Algorithm 6 does so by comparing the cost of all possible state transitions and therefore finds an optimal schedule.

Because $i \leq n_{0}, j \leq_{n_{N}}$ and $\delta \leq k \leq n_{0} n_{N}$, there are $O\left(n_{0}^{2} n_{N}^{2}\right)$ values of the state variables. Step 1 requires $O\left(n_{N} \log n_{N}\right)$. Step 2 requires constant time for each set of values of the state variables. Thus, the overall time complexity of Algorithm 6 is $O\left(n_{0}^{2} n_{N}^{2}\right)$.

Now, we consider problem $1 \mid p_{i} \leq p_{j} \Rightarrow d_{i} \leq$ $d_{j}, \Delta_{\max }\left(\pi^{*}\right) \leq k, p_{j}\left(a+b s_{j}\right) \mid \sum T_{j}$. From Lemmas 1, 2, and 3 , the maximum time disruption of jobs of $J_{0}$ is at most $k$, and jobs of $J_{N}$ before the last job of $J_{0}$ have the smallest due dates. Thus, we propose the following algorithm under the maximum time disruption constraint. (see Box 3 ).

Let $f(i, j, \delta)$ be minimum total tardiness value of a partial schedule for jobs $J_{1}, \ldots, J_{i}$ and $J_{n_{0}+1}, \ldots, J_{n_{0}+j}$, where the maximum time disruption is equal to $\delta$. The dynamic programming procedure can now be stated as follows.

Algorithm 9. Consider the following steps.

Step 1 (Initialization). Set $f(i, j, \delta)=0$ for $(i, j, \delta)=(0,0,0)$ and $f(i, j, \delta)=\infty$ for $(i, j, \delta) \neq(0,0,0), i=1, \ldots, n_{0}, j=$ $1, \ldots, n_{N}$, and $i=0, \ldots, k$.

Step 2 (Recurrence Relation).

$$
f(i, j, \delta)=\min \left\{\begin{array}{l}
f\left(i-1, j, \delta-P_{h}\right)+\max \left\{C_{i}-d_{i}, 0\right\} \\
f(i, j-1, \delta)+\max \left\{C_{n_{0}+j}-d_{n_{0}+j}, 0\right\},
\end{array}\right.
$$

where $P_{h}$ is the sum of actual processing time of the new jobs of $J_{N}$ between $J_{i-1}$ and $J_{i}$ and $C_{j}$ denotes the completion time of job $J_{j}$.
Step 3 (Optimal Solution). Calculate the optimal solution value $\min _{0 \leq \delta \leq k}\left\{f\left(n_{0}, n_{N}, \delta\right)\right\}$.

In the recurrence relation, the first term in the minimization corresponds to the case where the partial schedule ends with job $J_{i} \in J_{0}$. Because $J_{j}$ jobs of $J_{N}$ appear before job $J_{i}$ in such a partial schedule, the increase in the maximum time disruption is equal to $P_{h}$. The second term corresponds to the case where the partial schedule ends with job $J_{n_{0}+j} \in J_{N}$.

Similar to Example 7, by Algorithm 9, we have

(i) the maximum time disruption $k$ is in [1.7237, 25.7919]; the job sequence and the total tardiness cost are the same to Example;

(ii) the maximum time disruption $k$ is in $[0,1.7237)$, the job sequence is $\left[J_{2} \rightarrow J_{3} \rightarrow J_{1} \rightarrow J_{4} \rightarrow J_{6} \rightarrow J_{5}\right]$, and the total tardiness cost is 26.8007 .

Theorem 10. For the $1 \mid p_{i} \leq p_{j} \Rightarrow d_{i} \leq d_{j}, \Delta_{\max }\left(\pi^{*}\right) \leq$ $k, p_{j}\left(a+b s_{j}\right) \mid \sum T_{j}$ problem, Algorithm 9 finds an optimal schedule in $\mathrm{O}\left(n_{0} n_{N} C_{\max }+n_{N} \log n_{N}\right)$ time.

Proof. From Lemmas 1, 2, and 3, $\Delta \leq k$ means that the total actual processing time of the new jobs of $J_{N}$ before the last job of $J_{0}$ is at most $k$, and these are the jobs of $J_{N}$ with the smallest due dates. Hence, Algorithm 9 schedules the jobs according to the (EDD, EDD) property.

Because $i \leq n_{0}, j \leq n_{N}$ and $\delta \leq k<C_{\max }$, there are $O\left(n_{0} n_{N} C_{\max }\right)$ values of the state variables. Step 1 requires $O\left(n_{N} \log n_{N}\right)$. Step 2 requires constant time for each set of values of the state variables. Thus, the overall time complexity of Algorithm 9 is $O\left(n_{0} n_{N} C_{\max }+n_{N} \log n_{N}\right)$.

Now, we consider problem $1 \mid p_{i} \leq p_{j} \Rightarrow d_{i} \leq$ $d_{j}, \sum \Delta_{j}\left(\pi^{*}\right) \leq k, p_{j}\left(a+b s_{j}\right) \mid \sum T_{j}$. From Lemmas 1,2 , and 3 , there is the total time disruption of jobs of $J_{N}$ which is less than or equal to $k$ and can be sequenced before the last job of $J_{0}$, and these jobs have the smallest due dates. The following dynamic programming algorithm performs an optimal merging of jobs of $J_{0}$ and $J_{N}$ in a way similar to Algorithm 6. (see Box 4).

Let $f(i, j, \delta)$ be minimum total tardiness value of a partial schedule for jobs $J_{1}, \ldots, J_{i}$ and $J_{n_{0}+1}, \ldots, J_{n_{0}+j}$, where the total 
Input: Given $p_{j}, d_{j}$ for $j=1, \ldots, n$; $k$ and $\pi^{*}$, where $k<C_{\max }$ (see Lemma 1 ).

Indexing: Index the jobs of $J_{N}$ in the EDD order.

Box 3

Input: Given $p_{j}, d_{j}$ for $j=1, \ldots, n$; $k$ and $\pi^{*}$, where $k \leq n_{0} C_{\max }$.

Indexing: Index the jobs of $J_{N}$ in the EDD order.

Box 4

time disruption is equal to $\delta$. The dynamic programming procedure can now be stated as follows.

\section{Algorithm 11.}

Step 1 (Initialization). Set $f(i, j, \delta)=0$ for $(i, j, \delta)=(0,0,0)$ and $f(i, j, \delta)=\infty$ for $(i, j, \delta) \neq(0,0,0), i=1, \ldots, n_{0}, j=$ $1, \ldots, n_{N}$, and $i=0, \ldots, k$.

Step 2 (Recurrence Relation).

$f(i, j, \delta)=\min \left\{\begin{array}{l}f\left(i-1, j, \delta-\sum_{h=n_{0}+1}^{n_{0}+j} p_{[h]}\right)+\max \left\{C_{i}-d_{i}, 0\right\} ; \\ f(i, j-1, \delta)+\max \left\{C_{n_{0}+j}-d_{n_{0}+j}, 0\right\},\end{array}\right.$

where $p_{[h]}$ is the actual processing time of job $J_{h} ; C_{j}$ denotes the completion time of job $J_{j}$.

Step 3 (Optimal Solution). Calculate the optimal solution value $\min _{0 \leq \delta \leq k}\left\{f\left(n_{0}, n_{N}, \delta\right)\right\}$.

In the recurrence relation, the first term in the minimization corresponds to the case where the partial schedule ends with job $J_{i} \in J_{0}$. Because $J_{j}$ jobs of $J_{N}$ appear before job $J_{i}$ in such a partial schedule, the increase in total time disruption is equal to $\sum_{h=n_{0}+1}^{n_{0}+j} p_{[h]}$. The second term corresponds to the case where the partial schedule ends with job $J_{n_{0}+j} \in J_{N}$.

Theorem 12. For the $1 \mid p_{i} \leq p_{j} \Rightarrow d_{i} \leq d_{j}, \sum \Delta_{j}\left(\pi^{*}\right) \leq$ $k, p_{j}\left(a+b s_{j}\right) \mid \sum T_{j}$ problem, Algorithm 11 finds an optimal schedule in $\mathrm{O}\left(n_{0}^{2} n_{N} C_{\max }\right)$ time.

Proof. The proof of optimality of Algorithm 11 is similar to Theorem 8 regarding the time complexity. Because $i \leq n_{0}$, $j \leq n_{N}$, and $\delta \leq k \leq n_{0} C_{\max }$, there are $O\left(n_{0}^{2} n_{N} C_{\max }\right)$ values of the state variables. Step 1 requires $O\left(n_{N} \log n_{N}\right)$. Similar arguments to those in the proof of Theorem 8 show that the overall time complexity of Algorithm 11 is $O\left(n_{0}^{2} n_{N} C_{\max }\right)$.

\section{Conclusions}

In this paper, we studied the issue of rescheduling to allow the unexpected arrival of new jobs and took into account the effect of the disruption on a previously planned optimal schedule. The main contribution of this paper is that we develop the machine rescheduling scheduling problems agreeable job parameters under deterioration and disruption. Rescheduling means to schedule the jobs again, together with a set of new jobs. Deteriorating job means that the actual processing time of a job is an increasing function of its starting time. When the processing time and due date of jobs are agreeable, we considered some problems to minimize total tardiness under a limit on two disruption constraints: sequence disruption and time disruption. We proposed polynomial time algorithms or some dynamic programming algorithms for each problem. Future research may stimulate rescheduling models to mitigate the effects of the disruptions that occur frequently in manufacturing practice.

\section{Acknowledgments}

The authors would like to thank the authors of the references for enlightening them. This paper was supported by National Natural Science Foundation of China (71272085), Tianyuan Fund for Mathematics (11226237), the Humanities and Social Sciences Program of the Ministry of Education of China (12YJA630135), the Foundation of Chongqing Education Commission (KJ120624), and the Key Project Fundation of Chongqing Normal University (2011XLZ05).

\section{References}

[1] S. Browne and U. Yechiali, "Scheduling deteriorating jobs on a single processor," Operations Research, vol. 38, no. 3, pp. 495498, 1990.

[2] A. S. Kunnathur and S. K. Gupta, "Minimizing the makespan with late start penalties added to processing times in a single facility scheduling problem," European Journal of Operational Research, vol. 47, no. 1, pp. 56-64, 1990.

[3] G. Mosheiov, "Scheduling jobs under simple linear deterioration," Computers and Operations Research, vol. 21, no. 6, pp. 653659, 1994.

[4] T. C. E. Cheng, Q. Ding, and B. M. T. Lin, "A concise survey of scheduling with time-dependent processing times," European Journal of Operational Research, vol. 152, no. 1, pp. 1-13, 2004.

[5] S. Gawiejnowicz, Time-Dependent Scheduling, Springer, Berlin, Germany, 2008. 
[6] D. Biskup and J. Herrmann, "Single-machine scheduling against due dates with past-sequence-dependent setup times," European Journal of Operational Research, vol. 191, no. 2, pp. 587-592, 2008.

[7] J.-B. Wang and Q. Guo, "A due-date assignment problem with learning effect and deteriorating jobs," Applied Mathematical Modelling, vol. 34, no. 2, pp. 309-313, 2010.

[8] C. T. Ng, J.-B. Wang, T. C. E. Cheng, and L. L. Liu, "A branch-and-bound algorithm for solving a two-machine flow shop problem with deteriorating jobs," Computers \& Operations Research, vol. 37, no. 1, pp. 83-90, 2010.

[9] T. C. E. Cheng, W.-C. Lee, and C.-C. Wu, "Single-machine scheduling with deteriorating jobs and past-sequencedependent setup times," Applied Mathematical Modelling, vol. 35, no. 4, pp. 1861-1867, 2011.

[10] N. Raman, F. B. Talbot, and R. V. Rachamadugu, "Due date based scheduling in a general flexible manufacturing system," Journal of Operations Management, vol. 8, no. 2, pp. 115-132, 1989.

[11] L. K. Church and R. Uzsoy, "Analysis of periodic and eventdriven rescheduling policies in dynamic shops," International Journal of Computer Integrated Manufacturing, vol. 5, pp. 153163, 1992.

[12] A. K. Jain and H. A. Elmaraghy, "Production scheduling/rescheduling in flexible manufacturing," International Journal of Production Research, vol. 35, no. 1, pp. 281-309, 1997.

[13] G. E. Vieira, J. W. Herrmann, and E. Lin, "Rescheduling manufacturing systems: a framework of strategies, policies, and methods," Journal of Scheduling, vol. 6, no. 1, pp. 39-62, 2003.

[14] B. B. Yang, "Single machine rescheduling with new jobs arrivals and processing time compression," International Journal of Advanced Manufacturing Technology, vol. 34, no. 3-4, pp. 378384, 2007.

[15] N. G. Hall and C. N. Potts, "Rescheduling for new orders," Operations Research, vol. 52, no. 3, pp. 440-453, 2004.

[16] J. J. Yuan and Y. D. Mu, "Rescheduling with release dates to minimize makespan under a limit on the maximum sequence disruption," European Journal of Operational Research, vol. 182, no. 2, pp. 936-944, 2007.

[17] C. L. Zhao and H. Y. Tang, "Rescheduling problems with deteriorating jobs under disruptions," Applied Mathematical Modelling, vol. 34, no. 1, pp. 238-243, 2010.

[18] H. Hoogeveen, C. Lenté, and V. T'kindt, "Rescheduling for new orders on a single machine with setup times," European Journal of Operational Research, vol. 223, no. 1, pp. 40-46, 2012.

[19] J. Du and J. Y.-T. Leung, "Minimizing total tardiness on one machine is NP-hard," Mathematics of Operations Research, vol. 15, no. 3, pp. 483-495, 1990.

[20] R. L. Graham, E. L. Lawler, J. K. Lenstra, and A. H. G. Rinnooy Kan, "Optimization and approximation in deterministic sequencing and scheduling: a survey," Annals of Discrete Mathematics, vol. 5, pp. 287-326, 1979.

[21] A. Kononov and S. Gawiejnowicz, "NP-hard cases in scheduling deteriorating jobs on dedicated machines," Journal of the Operational Research Society, vol. 52, no. 6, pp. 708-717, 2001. 


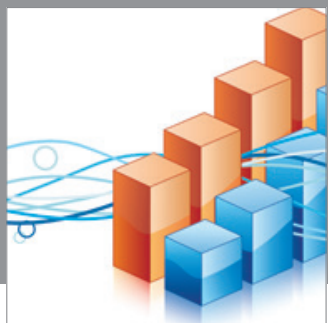

Advances in

Operations Research

mansans

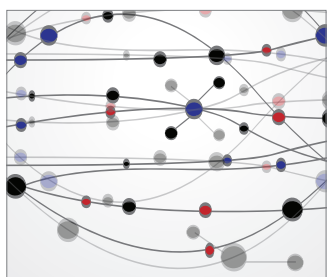

The Scientific World Journal
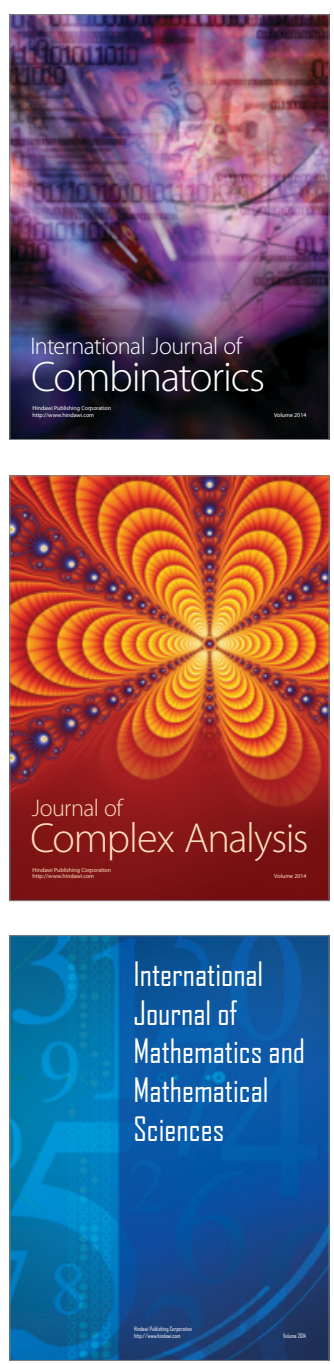
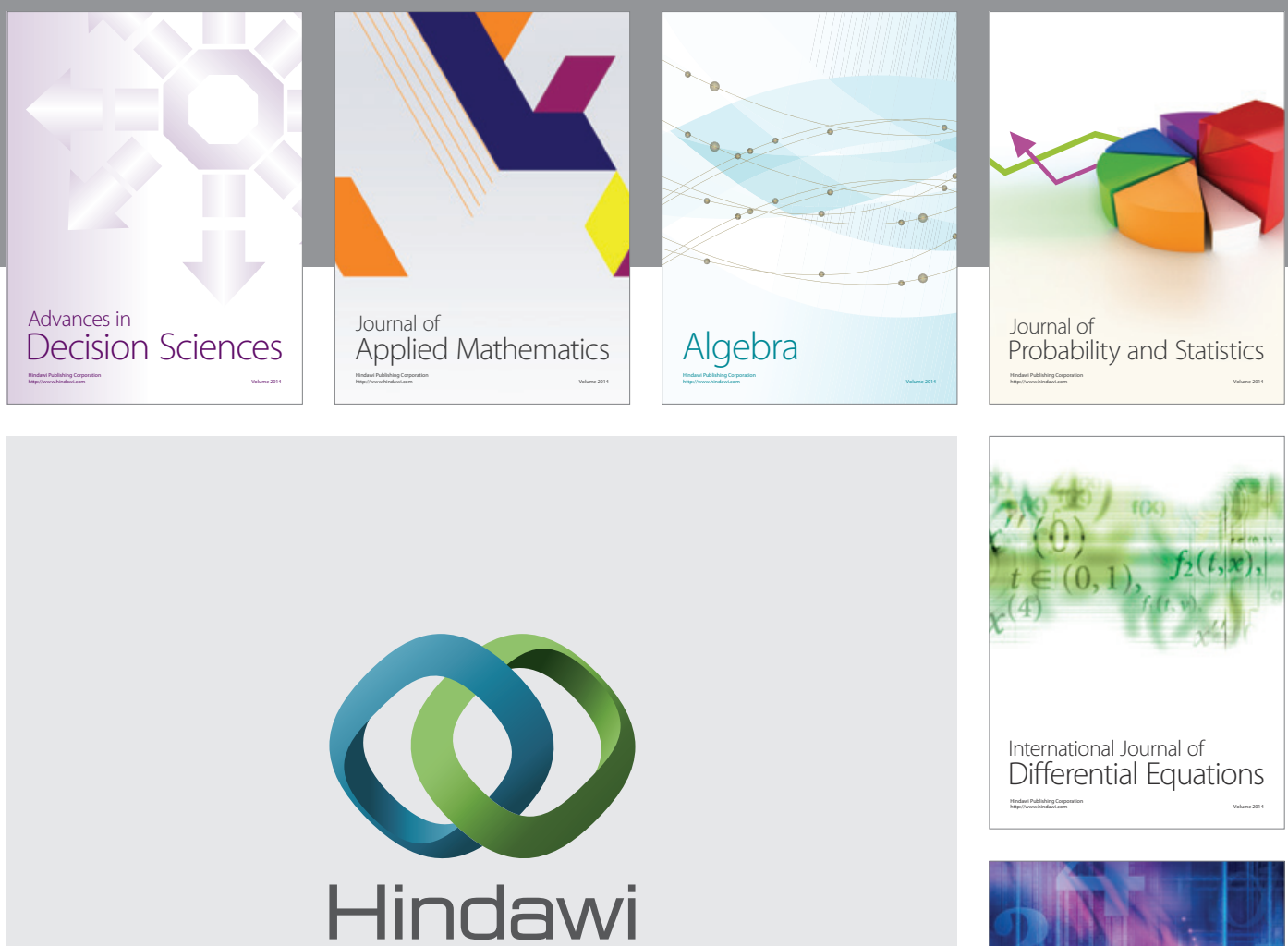

Submit your manuscripts at http://www.hindawi.com
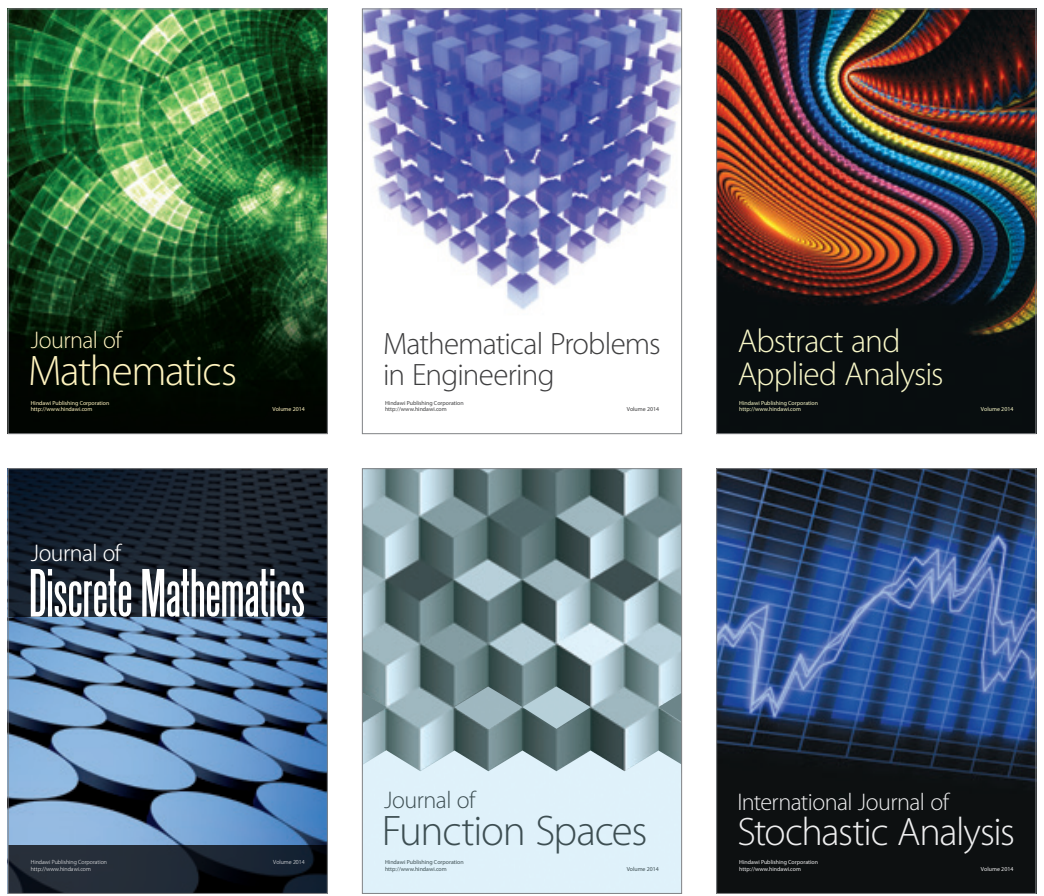

Journal of

Function Spaces

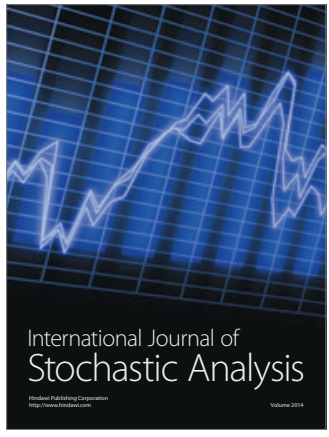

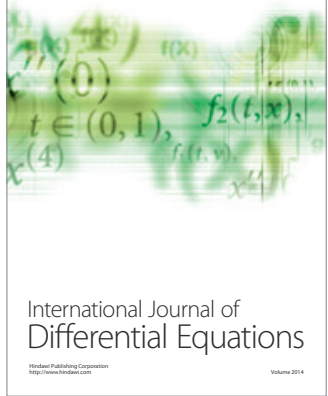
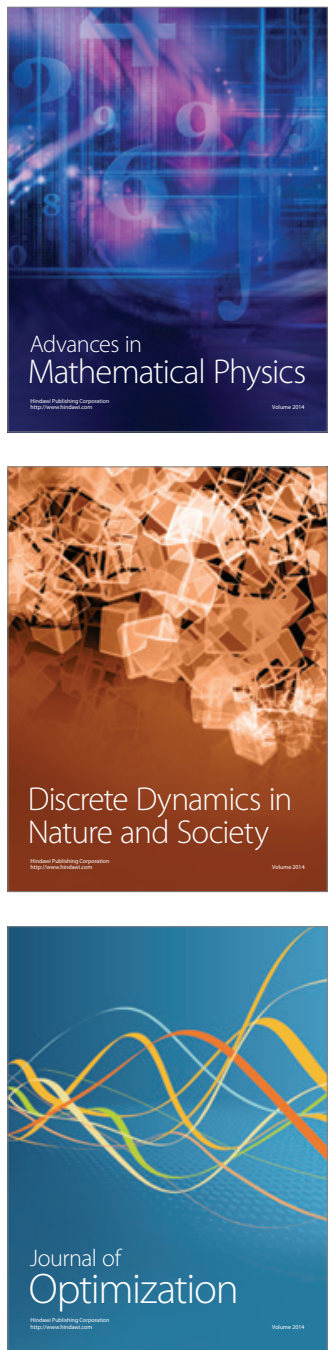